\title{
Zernike Phase Contrast Electron Microscopy: Observation of the Image Formation and Improvement of the Image Quality using Direct Detector
}

\author{
Kazuyoshi Murata ${ }^{1}$, Naoyuki Miyazaki ${ }^{1}$ and Kuniaki Nagayama ${ }^{2}$ \\ 1. National Institute for Physiological Sciences, Okazaki Japan. \\ 2. The Graduate University for Advanced Studies (SOKENDAI), Hayama, Japan.
}

Zernike phase contrast transmission electron microscopy (ZPC-TEM) is a technique to enhance the image contrast of the object using a thin-film phase plate, which has been practically achieved by Nagayama's group recently [1]. In ZPC-TEM, carbon thin film having a central small hole is inserted into the back focal plane of the objective lens, which delays the phase of the scattered electrons at $\pi / 2$ and enhance the phase contrast by interfering between scattered and unscattered electrons. This method is very useful for observation of unstained biological samples using cryo-EM. However, several factors including an electron loss by phase plate, and drift and charging of the phase plate limit higher resolution information at present [2]. Here, we applied a direct detector CMOS camera [3] to improve the ZPC image. The divided sub-frames revealed the image formation detail of ZPC-EM. The result was applied for a selection of COMS image frames. The ZPC images summed selected frames showed an improvement of the image quality.

For observation of the image formation of $\mathrm{ZPC}$, amorphous thin carbon films were imaged with conventional-EM and ZPC-EM, respectively, with an underfocus condition using JEM-2200FS equipped with an electron source of 200kV FEG (JEOL Inc.). The images were recorded on a direct detector CMOS camera, DE12 (Direct Electron LP) for $1 \mathrm{sec}$ exposure time divided with 25 serial frames of each $0.04 \mathrm{sec}$. The total electron dose was $\sim 30 \mathrm{e}^{-} / \AA^{2}$. As Zernike phase plate, it was used an amorphous thin carbon film at the thickness of $50 \mathrm{~nm}$ having a $500 \mathrm{~nm}$ central hole, which corresponded to $1 / 50 \mathrm{~nm}$ cut-on frequency. The each frame was processed by FFT and the resulting CTF profiles were compared. For Cryo-EM observation of biological specimen, ice-embedded sapovirus VLPs [4] on EM grid were inserted into the electron microscope using Gatan 914 cryo-specimen holder (Gatan Inc.). The images were recorded with the same procedures mentioned above with the electron dose of $\sim 20 \mathrm{e}^{-} / \AA^{2}$. The serial frames were aligned each other and summed after selecting the effective frames.

The CTF profiles taken by an underfocus condition using conventional EM and ZPC-EM were calculated in each frame and compared along the time course (Fig. 1). While the CTF profiles were constant in conventional EM frames, they were not in ZPC-EM frames where the CTF appeared from the low frequency side after $0.24 \mathrm{sec}$ exposure time (arrow in Fig. 1) and finally became a constant. These results suggest that it needs a pre-irradiation for several hundreds milliseconds to form and stabilize the ZPC images. It also means that the first several frames don't significantly contribute to the image formation.

The frame-divided imaging by direct detector was applied for an unstained biological specimen. Fig. 2A and B represent the ZPC images of ice-embedded sapovirus VLP summed with all and selected frames, respectively. In the selected summed image, the first 6 frames to $0.240 \mathrm{sec}$ were omitted. It looked similar to the all summed image, but the intensity of the power spectrum was slightly higher in the selected summed image than the all summed image. It suggests that the image quality (contrast) is improved by selecting the frames in ZPC-EM. 
Here we first present that the ZPC image is formed after several milliseconds of exposure. The contrast of ZPC cryoEM image enhanced by omitting the first several frames improves the image quality of the ice-embedded sapovirus VLP. This procedure would be believed to push the image resolution further in singe particle three-dimensional reconstruction of protein molecules using ZPC images in the future.

\section{References:}

[1] K Nagayama and R Danev Philos Trans R Soc Lond B Biol Sci. 363 (2008) 2153-2162.

[2] K Murata et al. Structure 18 (2010) 903-912.

[3] AF Brilot et al. J Struct Biol 177 (2012) 630-637.

[4] GS Hansman et al. FEBS Let 580 (2006) 4047-4050.
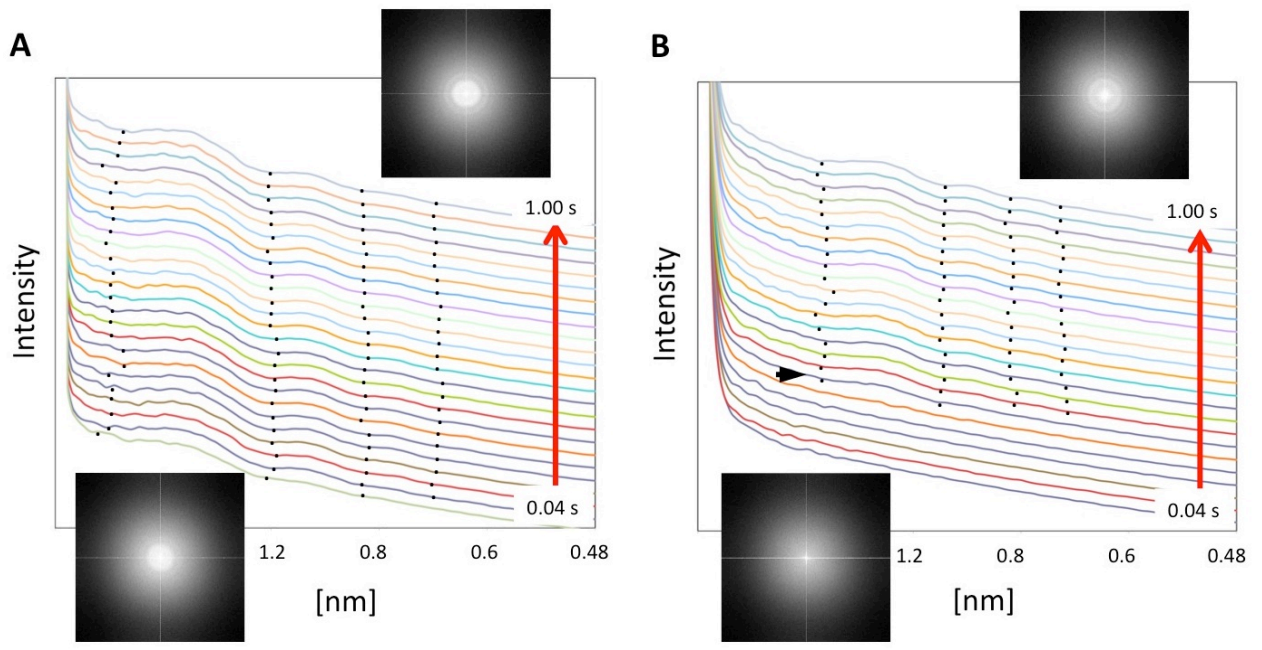

Figure 1. CTF profiles of amorphous carbon thin film in serial frames by conventional EM (A) and ZPC EM (B). Serial frames were recorded at every $0.04 \mathrm{sec}$ up to $1 \mathrm{sec}$ (Arrows). The CTF profile appears after 6 frames in ZPC image (arrow head) while it appears from the first frame in conventional EM. Dots shows zero nodes in CTF. CTF images of the first and last frames are inserted in the graphs.
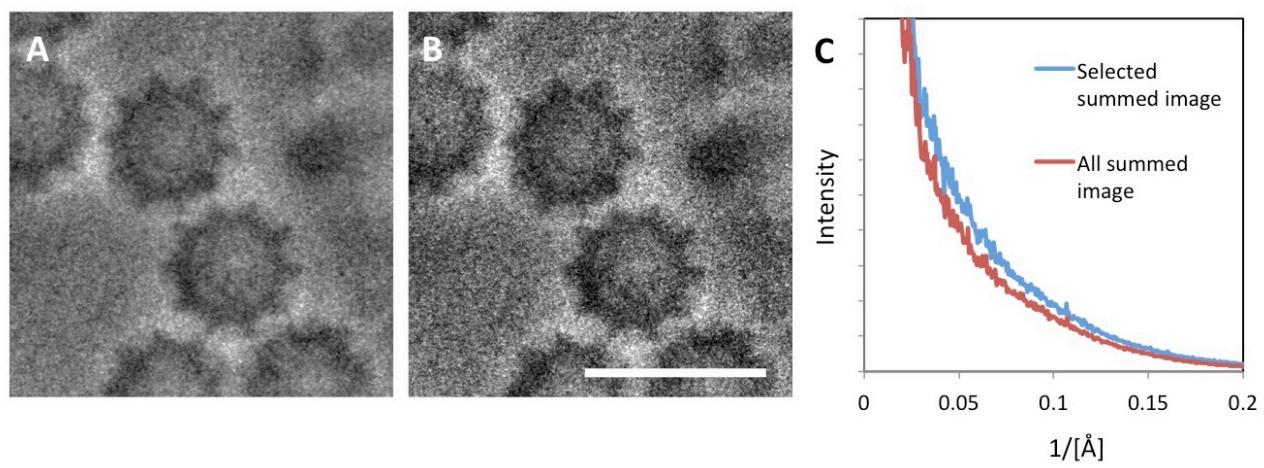

Figure 2. Ice-embedded sapovirus VLPs were recorded on the direct detector with serial frames of 0.04 sec each by ZPC-EM. The serial frames were aligned and summed with all (A) and selected (B: omitted the first 6 frames) frames, respectively. Scale $50 \mathrm{~nm}$. C) Power spectrum of all and selected summed images. 